\title{
Valeur et place de l'imagerie de la maladie de La Peyronie
}

\author{
E. SChOUMAN-Claeys* \\ Groupe Hospitalier Bichat Claude Bernard, Service de Radiologie, \\ 46 rue Henri Huchard, 75018 PARIS
}

\section{RESUME}

Cette présentation se propose, sur la base d'une revue de la littérature et de l'expérience de l'auteur (15 cas) de faire le point sur la place de l'imagerie (échographie et IRM) dans le cadre du bilan de maladie de La Peyronie.

L'échographie n'a dans cette étude objectivé que $62 \%$ des plaques perçues, chiffre qui se situe dans les normes de la littérature (59\% $96 \%)$. Les performances relativement limitées de l'échographie ont été notamment reliées à la fréquence peut-être insuffisante des sondes utilisées (7Mhz). L'IRM a permis de documenter l'atteinte dans $87 \%$ des cas (68$90 \%$ dans la littérature), sous la forme d'épaississement de l'albuginée périphérique, de plaques septales ou de strictions localisées. Une discrète prise de contraste a été objectivée dans $20 \%$ des cas. L'IRM n'a pas été contributive pour le diagnostic de calcification pas plus qu'elle n'a modifié la prise en charge thérapeutique. La discussion souligne les limites de l'IRM pour le diagnostic de plaque fibreuse infiltrante sans épaississement localisé de l'albuginée, la difficulté d'apprécier le caractère anormal d'une prise de contraste compte-tenu de la cinétique de diffusion du gadolinium dans les corps caverneux, les avantages et inconvénients d'explorations réalisés sous tumescence induite, les problèmes d'interprétation liés aux condures de la verge et enfin la variation des interprétations inter- et même intra-observateurs.
Il est donc proposé, après avoir souligné la valeur du gold standard clinique, de réserver les indications de l'imagerie, échographie et plus encore IRM, à des protocoles prospectifs ou à des difficultés diagnostiques particulières.

Mots-clés : Maladie de La Peyronie, imagerie, échographie, IRM

La maladie de La Peyronie résulte d'une atteinte de l'albuginée. Cette tunique qui circonscrit les corps caverneux présente alors des plaques, unique ou multiples, plus souvent localisées sur la face dorsale mais en fait de topographie ubiquitaire, notamment septale, avec parfois des prolongements intra caverneux. Il a toutefois été rapporté par certains [2] une relative prédilection pour le tiers moyen.

La lésion précoce est décrite comme un processus inflammatoire avec infiltration lympho-plasmocytaire périvasculaire [1], localisé à la couche de conjonctif aréolaire située à la partie profonde de l'albuginée. Ces plaques subissent une transformation fibreuse et peuvent se calcifier.

La ou les plaques ainsi formées peuvent être responsables, en érection, d'une déformation pénienne et de douleurs. Les patients peuvent par ailleurs présenter, à des degrés variables, une dysfonction érectile. Cette affection décri- 
te en 1743 par François de La Peyronie reste considérée comme idiopathique, encore que certaines théories évoquent une origine posttraumatique. Elle n'a ni marqueur ni traitement médical spécifiques. L'atteinte est acquise et n'est pas figée dans le temps, l'évolution pouvant se faire vers une aggravation, une stabilisation, rarement une amélioration.

Le diagnostic de la maladie est clinique, sur la reconnaissance à la palpation des plaques, aisément accessibles car très superficielles et de consistance indurée (induratio penis plasti$c a$ ). Quand la gêne fonctionnelle est importante se discute une approche chirurgicale. C'est également alors que l'on peut envisager d'utiliser l'imagerie pour compléter les données de l'examen clinique.

\section{IMAGERIE : LES DONNEES DE LA LITTERATURE}

Les différentes techniques d'imagerie qui utilisent respectivement les rayons $X$, les ultrasons ou encore la résonance magnétique du proton ont toutes été utilisées.

\section{Imagerie par $R X$}

Les clichés sans préparation, à rayons dits mous, peu pénétrants (au besoin sur un sénographe), adaptés aux parties molles, ont pour objectif de distinguer les zones fibreuses, plus denses que les tissus mous environnants ainsi que les zones calcifiées. Cette méthode est assez peu utilisée car elle est irradiante. Son rendement diagnostic serait par ailleurs très limité (4 patients sur 17 identifiés, soit une sensibilité de $24 \%$ [8].

\section{Les ultrasons}

L'aspect normal de l'albuginée est celui d'une bande hyperéchogène de délimitation imparfaite qui entoure les corps caverneux. Ces derniers sont de structure homogène et d'échogénéicité intermédiaire. Les tissus sous-cutanés sont quant à eux peu épais, d'épaisseur uniforme et d'échogénéicité un peu plus élevée que celle des corps caverneux.

La plaque mature apparait comme un épaississement plus ou moins hyperéchogène, loca- lisé, de l'albuginée. Les calcifications sont reconnues à leur échogénéicité très élevée (zones très brillantes) et à leur cône d'ombre acoustique. Les plaques récentes ont été décrites comme plus hypoéchogènes [4]; il est également possible d'observer une hypoéchogénéicité en périphérie de la plaque.

Les performances de l'échographie pour la détection des plaques sont diversement apprécié d'une série à l'autre.

En 1990 Pohar [8], dans sa série de 17 patients, rapportait une sensibilité de 59\% (10/17 patients) et relevait l'absence de corrélation entre palapation et détection échographique, pour conclure que l'échographie n'était pas fiable dans ce contexte et que le diagnostic devait probablement rester du seul domaine de l'approche clinique. Les résultats de l'étude de Vosshenrich [9], détectant 30 des 45 plaques palpables (sensibilité 67\%), sont assez proches.

A l'opposé d'autres, comme Hamm en 1986 [2], tiennent l'échographie comme une méthode idéale pour la documentation des plaques, leur caractérisation et leur suivi. Cette conclusion était portée sur une série de 49 patients chez lesquels 51 des 53 indurations palpées (96\%) avaient été décelées en échographie. Cette importante série localisait les plaques plus souvent sur le tiers moyen (55\% vs $24 \%$ pour le tiers distal et $21 \%$ pour la base), et, dans un plan transversal, plus souvent sur la face dorsale $(72 \%)$ et le septum (13\%).

L'échographie a par ailleurs pour intérêt de déceler d'éventuelles calcifications, qu'elle rapporte avec une prévalence assez variable : $27 \%-50 \%[9,8]$.

\section{L'IRM}

L'IRM a été proposée du fait de ses performances sur les tissus mous et de ses capacités d'acquisition multiplanaire.

L'albuginée normale est identifiée sous la forme d'une bande d'épaisseur uniforme de signal très faible, du fait d'une teneur très élevée en fibres collagènes, et ce, quelle que soit la séquence utilisée, alors que les tissus avoisinants ont tous un signal nettement plus élevé. Le contraste albuginée/corps caverneux 
est important sur les séquences pondérées en $\mathrm{T} 1$, et encore plus marqué sur les séquences pondérées en $\mathrm{T} 2$, du fait du signal des corps caverneux respectivement intermédiaire ou hyperintense, encore que cet hypersignal puisse en T2 n'intéresser que la région centrale [5]. Le fascia de Bück, qui délimite l'albuginée en superficie, a quant à lui un signal relativement élevé, contrastant également nettement avec l'albuginée.

La plaque prend l'aspect d'un épaississement de l'albuginée irrégulier; qui peut s'étendre en intracaverneux [9]. Certains auteurs [3,9] ont proposé de rechercher un rehaussement après injection de produit de contraste paramagnétique par voie intraveineuse pour apprécier la composante inflammatoire, mais ici encore les résultats des travaux publiés sont disparates.

Helweg [3] en 1992 rapporte une série de 28 patients, imagés sans tumescence induite et dont 18 présentaient une induration palpable et 10 de simples douleurs à l'érection et/ou une déviation. La technique semblait principalement utiliser de l'imagerie sagittale.

Limagerie T1 avait montré un épaississement localisé et des irrégularités de l'albuginée chez 20 des patients, dont les 18 porteurs d'anomalies à la palpation. Les plaques étaient plus souvent dorsales ( 14 cas) et septales ( 6 cas). Si l'imagerie T2 identifiait plus aisément les plaques, l'auteur ne la recommandait pas pour autant du fait de l'allongement du temps d'acquisition comparativement à une imagerie T1, en elle-même suffisamment contributive, ainsi que du fait des difficultés d'interprétation posées par les inhomogénéités spontanées de signal en T2 des corps caverneux (9 cas dans la série).

L'auteur relevait l'intérêt de l'IRM pour la détection de plaques non palpables (10 cas dont 2 décelés spontanément sans injection de produit de contraste paramagnétique et 5 des 8 autres cas identifiés après injection). Cette injection portait à $25 / 28(90 \%)$ le nombre de cas anormaux identifiés. Il soulignait également l'intérêt de l'IRM pour apprécier le caractère inflammatoire des plaques, sous la forme d'un rehaussement après injection, objectivé sur 10 des 18 patients injectés (55\%), dont 3 des 8 examens normaux avant injection et 7 des 10 examens d'emblée anormaux. Les images à l'appui montraient un réhaussement intéressant la portion sous-albuginéale du corps caverneux ou la zone profonde de l'albuginée. L'auteur concluait en faisant de l'IRM la technique de choix pour planifier le traitement et évaluer la réponse au traitement conservateur.

On doit à Vosshenrich [9] en 1994 une deuxième série importante portant sur 45 plaques palpables chez 34 patients, aux conclusions opposées. L'imagerie de base était ici réalisée sous tumescence induite (10 $\mu \mathrm{g}$ de PGE1), en sagittal, avec un complément en transversal chez 12 des 34 patients.

Cette série montre que l'IRM était plus sensible que l'échographie, tant pour déceler les plaques : $80 \%$ (36/45) vs $67 \%$ (30/45) que pour identifier les patients atteints de La Peyronie $68 \%$ (23/34) vs $59 \%$ (20/34). Un rehaussement après injection de Gadolinium était observé sur 4 des 36 plaques. En revanche aucune des calcifications observées en échographie (8/30 indurations palpables) n'était diagnostiquée en IRM.

L'auteur concluait que quand bien même l'IRM permettait une documentation précise des différentes plaques, elle n'apportait pas, tant pour le diagnostic de plaque fibreuse que le suivi des patients, d'avantage significatif par rapport au classique couple palpation échographie. L'auteur tenait enfin pour contestable l'information potentielle de l'IRM sur le caractère inflammatoire des plaques.

\section{NOTRE EXPERIENCE PERSONNELLE}

Notre expérience porte sur 15 cas explorés en IRM, âgés de $52 \pm 10$ ans ( $\min 28$, $\max 65$ ). Tous présentaient des plaques cliniquement palpables et aucun des antécédents de traumatisme ou des images évocatrices d'hématome; 13 de ces patients ont également été explorés en échographie.

Les échographies réalisées avec une sonde de $7.5 \%$ Mhz ont identifié des anomalies chez 8 des 13 patients $(62 \%)$, à type de plaques non calcifiées chez trois patients et de plaques cal- 
cifiées chez les cinq autres patients.

Les IRM ont été réalisées après injection de 10 $\mu \mathrm{g}$ de PGE1 sur une imageur Siemens SP42 $1 \mathrm{~T}$ ou un GE MR Max 0,5T. Le protocole associait une imagerie pré-injection en séquence pondérée $\mathrm{T} 1$ en sagittal et en axial puis un suivi en transversal en $\mathrm{T} 1$ après injection de gadolinium, combinant une acquisition immédiate (d'une durée de 2'20 à 4'50) suivie d'un minimum de trois séquences consécutives pour analyser la cinétique de diffusion du produit de contraste. Le plus souvent le protocole comprenait également une séquence axiale pondérée en T2.

Des anomalies ont été identifiées en IRM chez 13 des 15 patients (87\%) : épaississement de l'albuginée sous la forme d'irrégularités périphériques (11 cas), dont l'étendue apparaissait toujours plus importante qu'en clinique, plaque septale (3 cas, dont un associé à des anomalies de l'albuginée périphérique), striction localisée avec aspect en sablier (4 cas, présentant également des anomalies de l'albuginée périphérique). Comme en échographie, aucune plaque protrusive, intracaverneuse n'a été visualisée.

L'IRM a été considérée comme peu démonstrative par rapport à l'échographie dans 3 des 13 cas également explorés en échographie (23\%), par défaut d'identification de la ou des plaques pourtant cliniquement précisément localisées. Par ailleurs aucune des calcifications de l'albuginée périphérique ou septale décelées en échographie n'était identifiée en IRM. En revanche l'IRM a seule permis l'identification, chez un patient, de petites calcifications centro-caverneuses, aisément visualisées au sein du signal élevé du corps caverneux.

Un rehaussement focal après injection de Gadolinium considéré comme anormal a été constaté chez 3 des 15 patients (20\%), à type, dans un cas, de discret rehaussement en périphérie d'une plaque septale et dans deux cas de rehaussement également discret, curviliénaire, sous l'albuginée périphérique (l'un abservé dès le 2 ème passage, persistant sur le 3 ème et l'autre observé à partir du 3ème passage et également visible en T2). Un dernier cas n'a pas été retenu du fait du caractère transi- toire de l'hypersignal, non retrouvé sur l'incidence orthogonale.

$\mathrm{Au}$ total l'IRM nous a permis de documenter l'atteinte dans 13 des 15 cas. Si celle-ci apparaissait toujours assez largement étendue en IRM et notamment plus qu'en échographie, il n'était souvent pas possible d'identifier l'anomalie perçue cliniquement comme prédominante, ce qui rendait la corrélation précise avec l'échographie ou encore avec la clinique, plaque par plaque, hasardeuse sinon impossible. En aucun cas l'IRM n'a fait le diagnostic de calcification de l'albuginée. Enfin notre expérience nous a montré que la prise en charge du patient n'avait en aucune manière été modifiée pas les résultats de l'IRM.

\section{DISCUSSION}

\section{L'échographie}

L'échographie apparaît comme une méthode imparfaite, ne retrouvant globalement environ que $2 / 3$ des plaques palpées. Son intérêt essentiel est de déceler les calcifications, qui pourraient signer une atteinte évoluée, au potentiel de régression limité. Cette technique a également pour elle d'être combinée à la palpation, ce qui permet de relier directement l'anomalie palpée à l'anomalie visualisée.

On peut s'interroger sur les performances relativement limitées de l'échographie dans un contexte d'exploration superficielle, a priori simple. Une des explications tient à la nécessité d'une évaluation de la totalité de la circonférence du pénis. Ceci nécessite de multiplier les approches compte tenu des artefacts en bordure latérale de champ. Par ailleurs la recherche de calcifications, qui s'expriment classiquement par une surbrillance associée à un cône d'ombre, requiert, sur des plaques de topographie préférentiellement dorsales, un abord dorsal (le cône d'ombre pouvant être alors objectivé sur le corps caverneux plus postérieur). Cette incidence place le transducteur à proximité immédiate des zones anormales, avec d'une part le risque de compression par le poids de la sonde et, de l'autre, la gêne liée à la zone aveugle proximale, en amont de la focalisation la plus superficielle. L'utilisation d'une interface proposée par cer- 


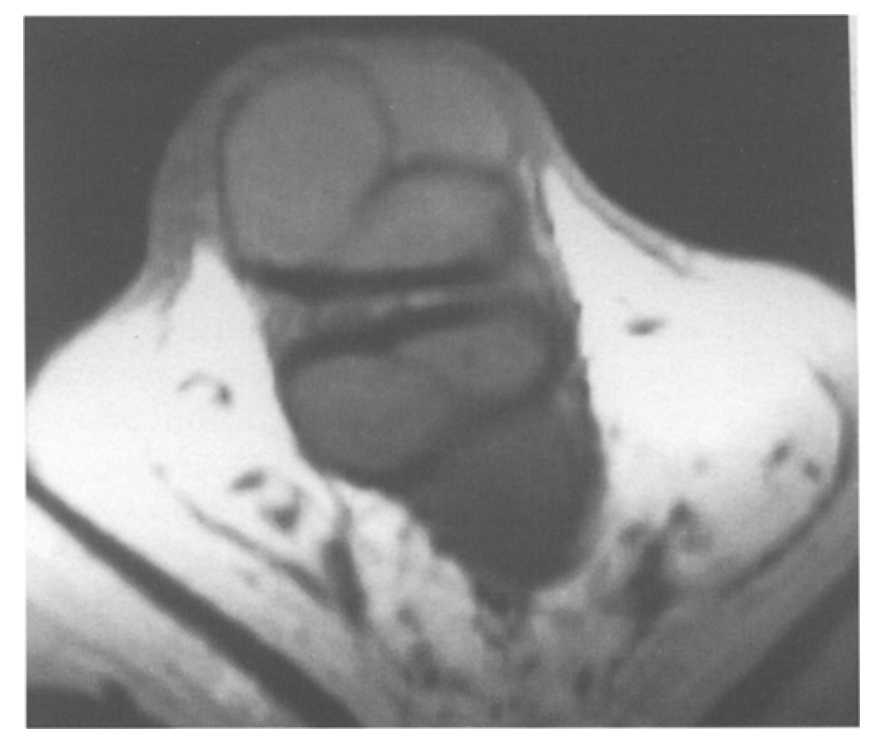

Cas $n^{\circ} 1$ : plaque non décelée en échographie, en hyposignal en IRM.

A la palpation plaque basale, bilatérale, à tendance circonférentielle. Echographie : aspect normal IRM 1T (après injection de $10 \mu \mathrm{g}$ de PGE1) en pondération $\mathrm{T} 1$ : coupe transversale, à proximité d'une courbure en recurvatum, permettant de ce fait de visualiser simultanément le tiers proximal et moyen de la portion mobile du penis. La plaque apparaît ici, par rapport à l'albuginée normale au signal d'un gris intermédiaire, sous la forme d'une zone d'hyposignal, quelque peu irrégulière, intéressant essentiellement la face dorsale.

Cas $n^{\circ} 3$ : plaque non visualisée en échographie, en hypersignal en IRM

A la palpation : deux petites plaques distales, l'une dorsale, l'autre latérale droite Echographie (A) : aspect normal IRM $1 \mathrm{~T}$ (B) ; coupe transversale pondérée en $\mathrm{T} 1$ : identification des anomalies cliniques sous la forme de zones en hypersignal modéré, infiltrant l'albuginée sans la déformer, de localisation à la fois latérale droite et ventrale.
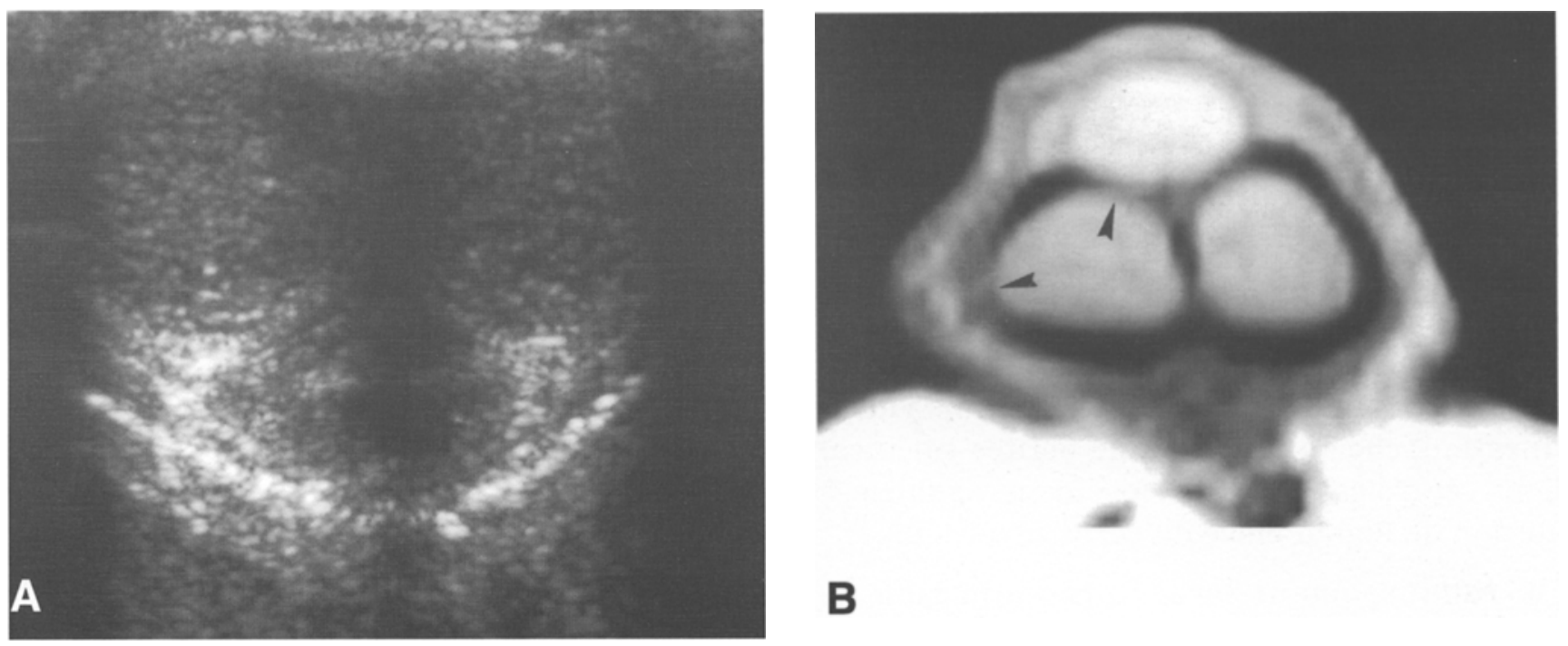
Cas $n^{\circ} 2$ : plaque aisément visualisée en échographie, calcifiées, d'aspect mixte, en hypo et en hypersignal en IRM.

A la palpation plaques distales dorsales. Echographie (A) : deux plaques nodulaires hyperéchogènes avec cône d'ombre au niveau de la face dorsale, à proximité du septum (têtes de flèche) IRM 0.5T (après injection de $10 \mu \mathrm{g}$ de PGE1, mais de faible efficacité) ; coupes transversales en pondération T1 à deux niveaux différents : - tiers proximal, peu modifié, où l'albuginée est d'aspect discrètement nodulaire avant injection de produit de contraste $(\mathbf{B})$, mais prend après injection $(\mathbf{C})$ un aspect normal, régulièrement hypointense; - coupe plus distale dans la zone d'aspect calcifié en échographie avant (D), puis après injection de Gadolinium (E); l'injection facilite quelque peu la délimitation des anomalies associant des zones nodulaires en hyposignal franc (tête de flèche) et des plages d'hypersignal infiltrant (flèche).
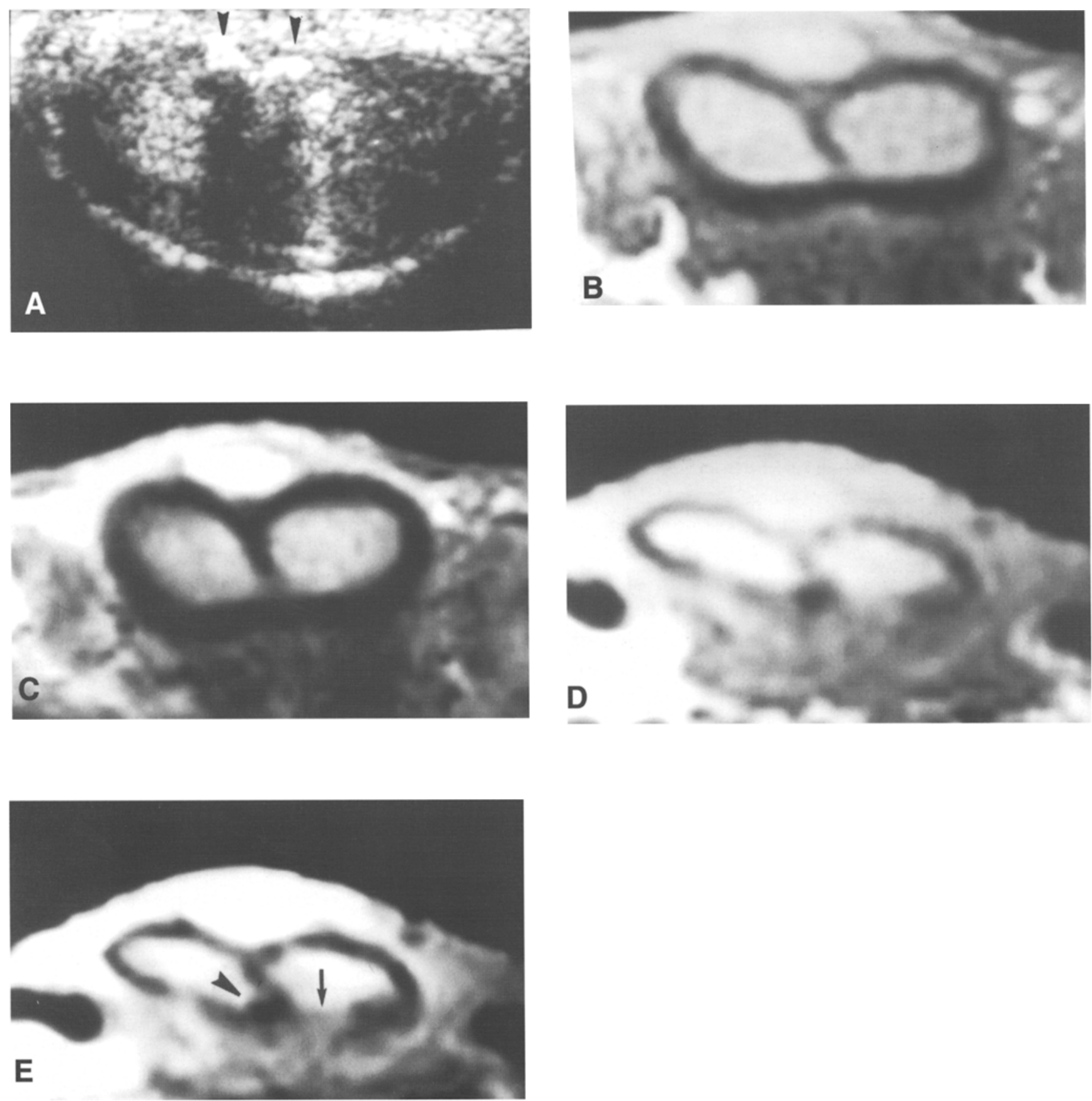
tains comme Hamm [2] tend à être abandonnée, car les matelas de composites gélifiés se dégradent rapidement, génèrent alors des artefacts et posent des problèmes d'hygiène. Les interfaces adaptées aux sondes sont quant à elles des accessoires coûteux pour une utilisation des plus restreintes. La réponse passe vraisemblablement par des sondes de très haute fréquence, maintenant commercialisées, qui offrent une excellente image dès les premiers mm. Ainsi Lencioni [7] rapporte la visualisation de plaques à $7 \mathrm{MHz}$ chez $64 \%$ (18/28) des patients et à $15 \mathrm{MHz}$ chez $90 \%$ (9/10) des cas considérés comme normaux à 7 $\mathrm{MHz}$.

\section{IRM}

Cette technique est intéressante dans son principe car elle permet une évaluation jointive et standardisée, analysable a posteriori. Le contraste entre albuginée et corps caverneux est par ailleurs meilleur qu'en échographie. Toutefois l'IRM pose différents problèmes.

Certains sont liées aux limites intrinsèques de la technique. Ainsi les calcifications ne contenant pas de protons apparaissent sous la forme d'une zone dépourvue de signal et ne se distinguent pas de la structure fibreuse de l'albuginée ou encore de plaques purement fibreuses, toutes deux très pauvres en signal. Reste que certaines plaques jeunes peuvent rendre plus intense le signal de l'albuginée et que la séméiologie décrite plus haut mérite alors d'être revue. On conçoit néanmoins qu'entre un signal d'une plaque jeune d'un gris plus clair que le gris-noir de l'albuginée normale, et la plaque très fibreuse ou calcifiée d'un noir à peine plus profond que le gris-noir de l'albuginée, le diagnostic d'une plaque infiltrante "plane", sans épaississement puisse rester difficile, sinon impossible en IRM. Cette technique ne fait donc aisément le diagnostic que des plaques épaississant de façon irrégulière l'albuginée, de celles qui sont responsables d'une striction des corps caverneux, avec en sagittal un aspect en sablier ou encore de celles à extension intracaverneuse (aspect que nous n'avons toutefois pas rencontré dans cette série).

Un avantage potentiel de l'IRM est sa sensibi- lité pour la détection des processus inflammatoires. Ces infiltrats ont un contenu élevé en eau (oedème) et peuvent être donc identifiés sur une imagerie T2. Surtout ils se rehaussent en imagerie $\mathrm{T} 1$ après injection de produit de contraste. Or on peut imaginer que la notion d'une inflammation ait une utilité clinique, incitant à l'utilisation de traitements ciblant spécifiquement cette composante et $a$ contrario conduisant à différer un éventuel geste chirurgical.

Toutefois, seule la série de Helweg [3] conclut à l'intérêt de l'IRM pour le diagnostic d'inflammation. Cette série est particulière par la fréquence élevée des rehaussements (10 sur 18 des cas injectés : 55\%) et s'oppose à leur rareté rapportée par Vosshenrich [9] (4 sur 36 plaques : $11 \%$ ) ou encore dans notre série (3 sur 15 patients : $20 \%$ ). Ces discordances pourraient être liées à une différence de technique d'acquisition : imagerie au stade de flaccidité dans la série de Helweg [3], sous tumescence dans la série de Vosshenrich [9] et la nôtre, avec la possibilité de masquer, par cette tumescence dont on sait qu'elle accélère le rehaussement du corps caverneux [6], les anomalies inflammatoires.

Toutefois on peut s'interroger sur la légitimité qu'il y a à attribuer de principe à une atteinte inflammatoire les rehaussements rapportés. En effet si le rehaussement des corps caverneux normaux a été décrit comme lent et centrifuge, s'étendant de l'artère caverneuse, de situation médio-caverneuse vers la périphérie [6], et de la base du corps caverneux vers son extrémité distale, des aspects différents ont été rapportés en cas d'insuffisance veineuse (rehaussement non centrifuge avec une importante prise de contraste périphérique) ou d'artériopathie (rehaussement retardé). L'interprétation des inhomogénéités de rehaussement se doit donc d'être particulièrement prudente, notamment chez des sujets chez qui, comme ici, peuvent également coexister des anomalies vasculaires. Les images de prise de contraste qualifiées d'anormales pourraient donc ne traduire qu'une hétérogénéité dans le remplissage des sinus caverneux et non une inflammation. Cette hypothèse est sous-tendue par le fait que nombre de ces aspects dits 
inflammatoires semblent, sur l'iconographie présentée par certains auteurs, localisés en intracaverneux, plus qu'en continuité directe avec la plaque à proprement parler. Sans doute serait-il donc nécessaire d'authentifier ces interprétations sur la base de comparaison avec l'imagerie $\mathrm{T} 2$, pour ne retenir comme indiscutables que les zones également anormales en 'T2, ou encore de s'aider comme dans notre pratique de l'analyse de la diffusion du rehaussement après injection sur des acquisitions sérielles, et cela en utilisant une imagerie transversale pour bien localiser le rehaussement.

Reste que l'hétérogénéité de population entre les patients de la série de Vosshenrich [9] ou la nôtre, qui tous présentaient des indurations palpables, et ceux de Helweg [3], dont certains ne présentaient pas de plaque palpable, est peut être l'indicateur dans cette dernière série de lésions plus jeunes, plus volontiers inflammatoires.

Enfin deux autres problèmes d'interprétation en IRM doivent être mentionnés. La possibilité d'erreurs d'interprétation liées à un positionnement de la verge non strictement orthogonal au plan de coupe, pouvant conduire à la construction d'»images pièges» en coupe sagittale. Ceci peut d'autant plus aisément se produire que ces patients présentent une déviation qui s'inscrit par elle même dans le cadre de l'affection, et qui peut se majorer en cours d'examen, sous tumescence induite. La réponse à ce problème passe dans l'avenir peut-être par des acquisitions tridimensionnelles permettant des reconstructions dans un autre plan de l'espace.

L'interprétation des images est enfin rendue difficile par la méconnaissance de l'épaisseur normale de l'albuginée. La littérature est en effet muette sur ce sujet, et l'épaisseur doit être interprétée à la fois en fonction du degré de tumescence (épaisseur physiologiquement plus importante au stade de flaccidité) et des courbures physiologiques, telle la plicature dorsale d'une verge maintenue dans cette position au cours de l'acquisition, avec pseudoépaissement de l'albuginée dans la concavité de la courbure.
On notera enfin, pour mémoire, l'aspect parfois trompeur de diminution globale du signal en profondeur, du fait de l'utilisation d'antennes de surface.

\section{CONCLUSION}

\section{Au total nous retiendrons que :}

1. la palpation reste un excellent moyen de diagnostic des plaques superficielles,

2. l'échographie est indiscutablement utile pour le diagnostic de calcification ; elle reste un examen non invasif et peu coûteux ; toutefois il ne s'agit pas nécessairement d'un examen simple à réaliser du fait notamment $d u$ caractère très superficiel des plaques,

3. l'IRM n'a pas jusqu'à présent clairement montré son bénéfice dans cette affection.

Elle ne fait pas le diagnostic de calcification, et pas ou assez mal le diagnostic de plaque fibreuse n'épaississant pas l'albuginée. Les images de rehaussement après injection intraveineuse de produit de contraste restent souvent d'interprétation difficile compte tenu d'une cinétique de diffusion un peu particulière au niveau des corps caverneux, et ce d'autant qu'il s'associe une atteinte vasculaire. Enfin la nécessité de faire un examen sous tumescence n'est pas totalement démontrée, quand bien même cette procédure qui permet une bonne distension de l'albuginée limite sans soute les faux positifs, avec la réserve des problèmes de coudure positionnelle. En revanche la tumescence accélérerait la captation du produit de contraste avec le risque de créer, en cas d'insuffisance vasculaire associée, de faux aspects inflammatoires.

Au total le diagnostic de la Maladie de La Peyronie reste donc du domaine clinique. L'IRM est, dans ce cadre, d'interprétation particulièrement délicate et notre expérience nous l'a montrée particulièrement soumise à d'importantes variations d'interprétation inter- et même intra-observateur. Il nous semble raisonnable, compte tenu par ailleurs de ce que l'IRM n'a pas dans ce contexte montré qu'elle apportait des éléments pesant 
dans la décision médicale et de son coût, de ne pas proposer cet examen en routine. Cette exploration devrait donc être réservée à des circonstances particulières : notamment protocoles prospectifs nécessitant une documentation précise de l'atteinte, diagnostic différentiels avec des lésions post-traumatiques des corps caverneux, plus qu'incertitudes cliniques quant à la caractérisation voire à l'étendue des lésions qui doivent alors bénéficier en première intention d'une échographie. Enfin si IRM il doit y avoir, il semble alors important pour en faciliter l'interprétation d'associer dans le même temps une exploration par échographie.

\section{RÉFÉRENCES}

1. CARSON C.C. : Peyronie's disease : newer diagnostic studies and surgical alternatives. Current Opinion in Urol, 1994,4 : 180-185

2. HAMM B., FRIEDRICH M., KELAMI A. : Ultrasound Imaging in Peyronie Disease. Urology, 1986, 28: $540-545$

3. HELWEG G., JUDMAIER W., BUCHBERGER W. et al : Peyronie's Disease: MR findings in 28 patients. AJR 1992, 158: 1261-1264

4. HERBENER T.E., SEFTEL A.D., NEHRA A., GODSTEIN I. : Penile Ultrasound. Sem Urol, 1994, 12: 320-332

5. HRICAK H., MAROTTI M., GILBERT T.J., LUE T.F., WETZEL L.H., MCANINCH J.W., et al : Normal penile anatomy and abnormal penile conditions: evaluation with MR Imaging, Radiology 1988, 169 : 683-690

6. KANEKO K., DE MOUY E.H., LEE B.E. : Sequential Contrast-enhanced MR Imaging of the Penis. Radiology 1994; 191:75-77

7. LENCIONI R.A., PAOLOCCHI A., MORELLI G., PETRUCCI R., PINTO F., BARTOLOZZI C. :Peyronie disease: evaluation with dynamic High-Frequency Sonography and Power Doppler Sonography, Radiology 1995, 197 : 286-287

8. POHAR S.S., JACKSON F.I., GLAZEBROOK G..A. : Ultrasonography and radiology in the diagnosis of Peyronie's disease. Can Assoc Radiol J, 1990, 41 : 369-371

9. VOSSHENRICH R., SCHROEDER-PRINTZEN I., WEIDNER W., FISCHER U., FUNKE M., RINGERT R.H. : Value of Magnetic Resonance Rmaging in Patients with Penile Induration (Peyronie's disease). J Urol 1995, 153 : 112-1125

\section{ABSTRACT} Sonography and MRI for investigation of
Peyronie's disease.

\section{E. Schouman-Claeys}

This presentation proposes, based on a review of the literature and the author's experience (15 cases), to define the role of present imaging studies (sonography and MRI) for investigation of Peyronie's disease.

In this study sonography only displayed $62 \%$ of the palpable plaques, a figure in line with the literature (59-96\%). MRI documented the lesion in $87 \%$ of the cases $(68-90 \%$ in the literature), as thickening of the peripheral albuginea, septal plaques or localized striction. A minor localized enhancement Gadolinium injection was displayed in $20 \%$ of the cases. MRI did not identify calcifications, nor did it modify treatment.

The discussion underlines the limits of MRI for diagnosis of fibrotic infiltrating plaques without localised thickening of the tunica albuginea, the difficulties to appreciate abnormal uptake of contrast medium, taking into consideration the diffusion kinetics of Gadolinium in the corpus cavernosum, the advantages and disadvantages of exploration during induced tumescence, the problems of interpretation due to penis curvature and finally the intra and inter observers interpretation variations.

Considering the value of the clinical gold standard, it is proposed to reserve sonography and especially MRI to prospective protocols or to particular diagnostic difficulties.

Key words: La Peyronie's disease, sonography, MRI 